\title{
THE ACOUSTIC EMISSION RESPONSE OF SNOW
}

\author{
By William F. St Lawrence \\ (U.S. Army Cold Regions Research and Engineering Laboratory, Hanover, New \\ Hampshire 03755, U.S.A.)
}

\begin{abstract}
In this work a model of the ultrasonic acoustic emission response in snow is developed. The model derived considers the acoustic emission response in snow as a function of stress and strain. It is suggested that the acoustic emission activity in snow is a quantitative indication of the creep rupture taking place in the material. The governing differential equation is developed; an example is then presented that considers the applicability of this equation to the release of certain types of avalanche.

Résumé. L'émission acoustique de la neige. Dans ce travail on développe un modèle de l'émission acoustique ultrasonore dans la neige. Le modèle considère l'émission acoustique comme une fonction de la contrainte et de la déformation. On suggère que l'activité émissive acoustique dans la neige est une indication quantitative des ruptures dues à la fluage qui se produisent dans le matériau. On développe l'équation différentielle qui régit le phénomène: on présente alors un exemple qui envisage l'applicabilité de cette équation au déclenchement de certains types d'avalanches.

Zusammenfassung. Die Schallemission von Schnee. In dieser Arbeit wird ein Modell für die Emission von Überschallwellen aus Schnee entwickelt. Das Modell betrachtet diese akustische Emission als eine Funktion von Spannung und Verformung. Es wird angenommen, dass die Schallemission von Schnee eine quantitative Anzeige für die Kriechbrüche sind, die in dem Material stattfinden. Die massgebliche Differentialgleichung wird hergeleitet; an einem Beispiel wird dann die Anwendbarkeit dieser Gleichung auf den Abgang bestimmter Lawinentypen untersucht.
\end{abstract}

\section{INTRODUCTION}

Over the past several years a number of investigations have been conducted that consider the nature of the seismic and acoustic waves that emanate from snow. Such investigations have been carried out both in the laboratory and in the field on avalanche-prone snow slopes. These studies have examined stress-induced noise in snow over a frequency range that varies from a few hertz to several hundred thousand hertz. Although the results obtained from the various studies of the acoustic emission phenomena in snow are still only preliminary, the indications are that seismo-acoustic emissions are related to slope stability or instability. However, the precise relationship between emissions and stability is not altogether clear.

Investigators have obtained field results (Gubler, 1979; Sommerfeld, 1977; St Lawrence and Bradley, 1977) that indicate that an increase in the seismo-acoustic activity from snow slopes is associated with an increase in avalanche activity. However, it has also been observed (Bowles and St Lawrence, I 977) that quiescent periods may also be associated with snow-slope instability. Thus we appear to be faced with the dilemma of apparently contradictory observations indicating the same result. This problem is similar in nature to the problem encountered in earthquake prediction, when at times swarms of small earthquakes may precede a large earthquake and at other times there is no such indication. Our inability to interpret correctly such contradictory evidence is an indication that we do not fully understand the nature of the system we are dealing with.

To understand the microseismic and acoustic emission activity from snow it is necessary to consider the nature of subcritical crack growth* (creep rupture). To do this we make the assumption that the signals detected from snow originate from fracture events. We also assume that the low-frequency signals detected are from relatively large fracture events and that the high-frequency signals are from relatively small fracture events. For example, the seismic signal associated with a fracture related to slab-avalanche release may be detectable at a distance on the order of hundreds of meters, while the ultrasonic emission associated with \footnotetext{
* We define subcritical crack growth as any fracture that occurs which does not result in a total disintegration
of the material system.
} 
grain-bond failure or even partial grain-bond failure may be detected at a distance of only a few centimeters. In small-scale events, such as bond failure, there are no detectable lowfrequency emissions.

In terms of the concept of creep rupture that we examine in this work, we present the following hypothesis to explain the apparently contradictory evidence we obtain from our field observations. In the instance where no premonitory signals are associated with catastrophic failure, we are dealing with a case of brittle failure. In this instance, a fracture that is initiated continues to propagate until complete failure of the material system takes place. In the second instance, detectable seismic (low-frequency) radiation may be associated with an instability. In this case fractures are arrested prior to catastrophic failure. This can be considered a case of marginal stability in that such fractures can lead to a major failure but, if they do not, they locally reduce the strain energy available for fracture.

In the third case we detect only high-frequency radiation, indicating that fracture is taking place on a scale which involves individual grains. In this instance local fracture is a quasistable mechanism of energy dissipation, and only under certain conditions will it lead to a catastrophic material failure.

This third type of ultrasonic emission which is associated with localized failure, is examined here. Specifically, we consider the creep rupture of snow as we interpret it from the presence of ultrasonic acoustic emissions. In particular, we develop a model to describe the acoustic emission response that is observed in snow.

\section{Acoustic Emissions in SNOW}

For the purpose of this paper, we define acoustic emissions to mean the ultrasonic emissions detected from snow subjected to mechanical loading. Descriptions of these emissions and the types of equipment used to detect them have been given in several papers (St Lawrence and Bradley, [1975]; St Lawrence, unpublished), the source of the emissions has not been observed and so it can only be inferred from observations of other materials.

We propose that the acoustic emissions are the result of small fractures that take place at grain boundaries or possibly through the ice grains. Gold (1960) has noted that the acoustic emissions from dense polycrystalline ice are a result of intra- and intergranular fracture. Since snow is a low-density form of ice it seems reasonable to assume that the source mechanisms are the same in both materials. Also, if we consider that the frequency of the acoustic source is a function of the time over which the fracture takes place, extrapolating from low frequency to high frequency suggests that the source of the ultrasonic emissions is a brief cracking event. This is suggested because the low-frequency events in snow are related to fracture (St Lawrence and Bradley, 1977). Accepting the above assumption allows us to speculate that the acoustic emissions are a quantitative measure of the creep-rupture activity in deforming snow.

Since it is observed that the acoustic emission activity exhibits well-defined patterns in the field and also in laboratory tests, it seems reasonable to attempt to describe its behavior. This is also important because the acoustic activity is functionally related to the deformation of snow (St Lawrence, unpublished) and represents a means other than viscous and plastic flow mechanisms by which strain energy is dissipated.

In attempting to describe the acoustic emission function for snow, we developed a model that represents the acoustic emission as a function of strain alone (St Lawrence, I979, unpublished). Here we extend our previous analysis and present it in a more general form.

Functionally we state that the acoustic emission response, or alternatively the creep rupture, can be written in the form:

$$
\mathcal{N}=\mathcal{N}(\sigma, \epsilon, T, s),
$$


where $\mathcal{N}$ is the number of acoustic emissions per unit volume of material, $\sigma$ is stress, $\epsilon$ is strain, $T$ is temperature, and $s$ is a variable related to the structure or texture of the snow. The textural variable is intended to include such snow properties as the snow type and the degree of sintering.

Working within the realm of available data, we confine our analysis to constant temperatures and assume that within the time frame of our experiments the texture of the snow is constant. This latter assumption imposes a time constraint on the theory, since application of external forces induces metamorphic changes. The total differential of Equation (I) with temperature and structure held constant is:

$$
\mathrm{d} \mathcal{N}=\frac{\partial \mathcal{N}}{\partial \sigma} \mathrm{d} \sigma+\frac{\partial \mathcal{N}}{\partial \epsilon} \mathrm{d} \epsilon
$$

Equation (2) now represents a point from which we can determine specifically the acoustic emission response for a given load condition.

In considering the specific functions in Equation (2) (i.e. $\partial \mathcal{N} / \partial \sigma$ and $\partial \mathcal{N} / \partial \epsilon$ ) we have chosen for reasons of simplification to develop the model from constant strain-rate data in uniaxial tension. This is done because snow subjected to tensional loadings shows no indication of work hardening;* this simplifies the analysis. For constant strain-rate, Equation (2) can be differentiated with respect to strain to obtain

$$
\frac{\mathrm{d} \mathcal{N}}{\mathrm{d} \epsilon}=\frac{\partial \mathcal{N}}{\partial \sigma} \frac{\mathrm{d} \sigma}{\mathrm{d} \epsilon}+\frac{\partial \mathcal{N}}{\partial \epsilon}
$$

Equation (3) is the total derivative of the acoustic emission response with respect to strain. To obtain the solution to Equation (3), it is necessary to determine the functional form of the partial derivative terms (i.e. $\partial \mathcal{N} / \partial \sigma, \partial \mathcal{N} / \partial \epsilon)$.

\section{STRAIN-INDUCED ACOUSTIC EMISSION AGTIVITY IN SNOW}

As a means of examining the nature of the strain-induced acoustic emission response $\partial \mathcal{N} / \partial \epsilon$ from a constant strain-rate experiment, the acoustic activity is examined after the point on the stress-strain curve where $\mathrm{d} \sigma / \mathrm{d} \epsilon$ is less than or equal to zero. This is why the tension data were chosen. For $\mathrm{d} \sigma / \mathrm{d} \epsilon$ equal to zero, Equation (3) reduces to

$$
\frac{\mathrm{d} \mathcal{N}}{\mathrm{d} \epsilon}=\frac{\partial \mathcal{N}}{\partial \epsilon}
$$

so that the total derivative is equal to the partial derivative.

The form of the strain-induced acoustic activity for snow is (St Lawrence, 1979)

$$
\frac{\partial \mathcal{N}}{\partial \epsilon}=\mathcal{N} c \exp (-\phi \epsilon)
$$

where $c$ and $\phi$ are experimentally determined constants. Equation (5) indicates that the rate of acoustic activity is proportional to the total activity that has occurred up to some strain $\epsilon$. The exponential term in Equation (5) serves to limit the rate of geometric increase in the emissions. Rearranging Equation (5) and putting it in logarithmic form gives

$$
\ln \left[\frac{\mathrm{I}}{\mathcal{N}} \frac{\partial \mathcal{N}}{\partial \epsilon}\right]=\ln (c)-\phi \epsilon
$$

From Equation (6) a linear least-squares analysis of the data can be used to compare it with the expected result and to determine the value of the constants $c$ and $\phi$. Figure $\mathrm{I}$ is a plot of

\footnotetext{
* For our purposes we define work hardening, in a constant strain-rate test, as a further increase in the stress with strain after significant yielding has taken place.
} 
experimental data arranged in a form consistent with Equation (6) for a snow sample subjected to a strain-rate of $1.67 \times 10^{-5} \mathrm{~s}^{-1}$. The correlation coefficient for these data is -0.999 , which indicates good agreement between the data and the result expected from Equation (5). Table I gives the values of $c, \phi$, and $r$ associated with the six tests associated with this analysis. As can be determined by examining the values of the correlation coefficients in Table I, the agreement between the data and Equation (5) is generally good.

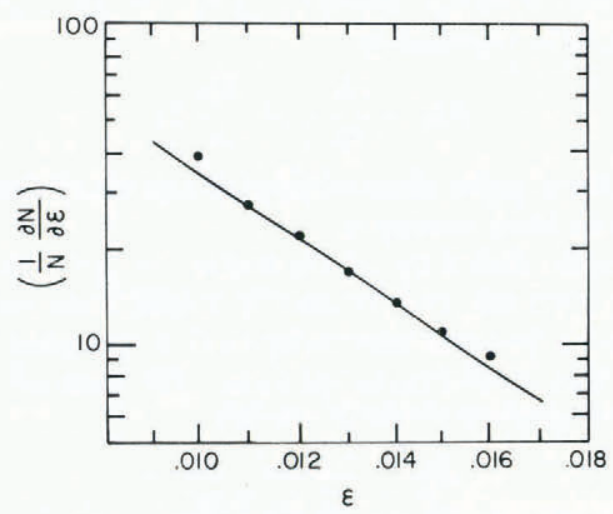

Fig. I. Logarithm of $(I / \mathcal{N})(\partial \mathcal{N} / \partial \epsilon)$ plotted versus strain for a snow sample strained at a constant tensional rate of $16.7 \times 10^{-6} \mathrm{~s}^{-1}$ at $-10^{\circ} \mathrm{C}$.

TABle I. Values of the constants and correlation COEFFicients associated with EQUATIONS (5) AND (8)

\begin{tabular}{rrrrrrr}
$\begin{array}{c}\text { Strain-rate } \dot{\epsilon} \\
\mathbf{s}^{-1}\end{array}$ & $c$ & $\phi$ & \multicolumn{1}{c}{$\boldsymbol{c}$} & \multicolumn{1}{c}{$A$} & $n$ & $r$ \\
$8.33 \times 10^{-6}$ & 28 & 50 & -0.958 & $2.74 \times 10^{-12}$ & 3.346 & 0.9282 \\
$11.1 \times 10^{-6}$ & 118 & 133 & -0.987 & $2.97 \times 10^{-9}$ & 2.643 & 0.9947 \\
$13.9 \times 10^{-6}$ & 243 & 183 & -0.995 & $1.06 \times 10^{-8}$ & 2.506 & 0.9841 \\
$16.7 \times 10^{-6}$ & 347 & 232 & -0.999 & $6.34 \times 10^{-10}$ & 2.744 & 0.9955 \\
$19.4 \times 10^{-6}$ & 480 & 233 & -0.979 & $1.06 \times 10^{-10}$ & 2.887 & 0.9916 \\
$22.2 \times 10^{-6}$ & 123 & 147 & -0.986 & $3.58 \times 10^{-10}$ & 2.852 & 0.9897
\end{tabular}

\section{Stress-Induced ACoustic AGTIVITY IN SNOW}

In determining the form of $\partial \mathcal{N} / \partial \epsilon$ for Equation (3), a conceptual model was developed (St Lawrence, r 979). In determining the stress-induced emission activity, we choose to use an empirical relation. Gold ( $1972[\mathrm{a}],[\mathrm{b}]$ ) indicates that for columnar-grained ice, the crack density as a function of stress is reasonably well represented by an equation of the form

$$
\mathcal{N}^{\prime}=A^{\prime}\left(\sigma-\sigma_{0}\right)^{i},
$$

where $\mathcal{N}^{\prime}$ is the number of cracks observed in a given area of sample, $\sigma$ is the applied stress, and $\sigma_{0}, A^{\prime}$, and $i$ are constants. The equation used here to represent the partial derivative of the acoustic activity with respect to stress is

$$
\frac{\partial \mathcal{N}}{\partial \sigma}=A\left(\sigma-\sigma_{0}\right)^{n}
$$

To examine the applicability of Equation (8), Equation (3) was solved for $\partial \mathcal{N} / \partial \sigma$ from a number of tension tests conducted at several different strain-rates. The values of the constants $A$ and $n$ for each test are tabulated in Table I along with the correlation coefficients determined for each test. Generally, Equation (8) produced a reasonable representation of the data. 
In all cases, the value of the constant $\sigma_{0}$ in the equation was taken to be zero. This assumption implies that there will be stress-induced emissions at very low stresses and that the snow has not been subjected to any prior stress. For the analysis presented here, the assumption that $\sigma_{0}$ is zero is adequate. Figure 2 is a $\log -\log$ plot of the experimental data from a constant strain-rate test.

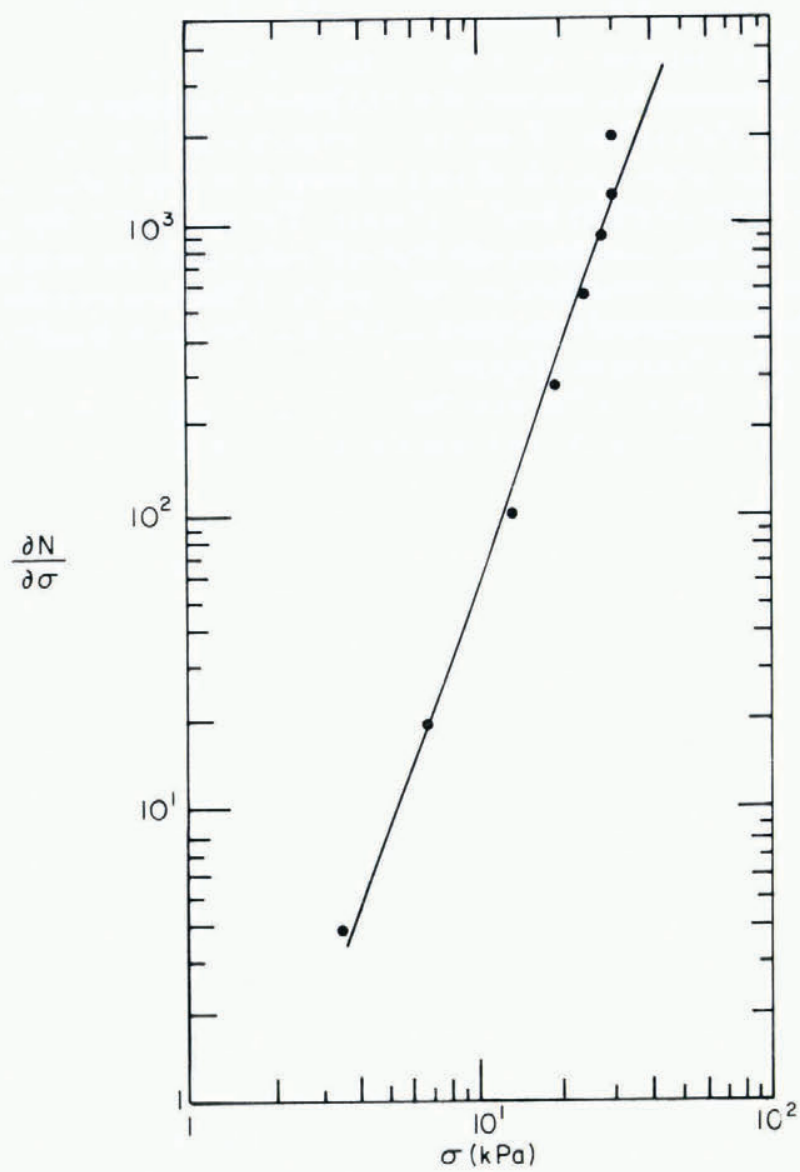

Fig. 2. Logarithm of $\partial \mathcal{N} / \partial \sigma$ versus the logarithm of $\sigma$ for a snow sample strained at a constant tensional rate of $16.7 \times 10^{-6} \mathrm{~s}^{-1}$ at $-I O^{\circ} \mathrm{C}$.

The PATtern OF Acoustic EMISSIONS

Having found an empirical and phenomenological description of the various partialderivative terms in Equation (3) allows us to solve that equation to obtain an integrated result. Rearranging Equation (3) and substituting the appropriate terms from Equations (5) and (8) gives

$$
\frac{\mathrm{d} \mathcal{N}}{\mathrm{d} \epsilon}-\mathcal{N} c \exp (-\phi \epsilon)=\frac{\partial \mathcal{N}}{\partial \sigma} \frac{\mathrm{d} \sigma}{\mathrm{d} \epsilon} .
$$

Noting that the stress $\sigma$ can be related to the strain $\epsilon$ through the constitutive relation for the material, allows us to reduce Equation (9) to an ordinary differential equation of the form 


$$
\frac{\mathrm{d} \mathcal{N}}{\mathrm{d} \epsilon}-\mathrm{f}(\epsilon) \mathcal{N}=\mathrm{p}(\epsilon),
$$

where $f(\epsilon)$ and $p(\epsilon)$ are functions of strain. The solution of Equation (9) is:

$$
\mathcal{N}=\left[K+\int \exp \left\{\frac{c}{\phi} \exp (-\phi \epsilon)\right\} \frac{\partial \mathcal{N}}{\partial \sigma}\left(\frac{\mathrm{d} \sigma}{\mathrm{d} \epsilon}\right) \mathrm{d} \epsilon\right] \exp \left\{-\frac{c}{\phi} \exp (-\phi \epsilon)\right\},
$$

where $\mathcal{N}$ is the number of acoustic emissions per unit volume and $K$ is a constant of integration associated with the homogeneous solution of the differential equation. Physically the constant $K$ can be interpreted as the number of potential sites from which acoustic emissions (fractures) will emanate at very low stress such as when the integral in Equation (I I) is zero. This type of low-stress rupture is described by Gold (I972[a]). The integral in Equation (I I) represents the additional number of acoustic emissions that will occur with an increase in the stress level. The term in brackets can be considered as the number of fracture sites that have the potential for activation and the exponential multiplier acts to describe the manner in which the acoustic sources are activated as a function of strain.

To demonstrate the nature and form of Equation ( 1 i ) we present the following numerical example. The data in this example are obtained from a constant strain-rate uniaxial tension test at $-10^{\circ} \mathrm{C}$.

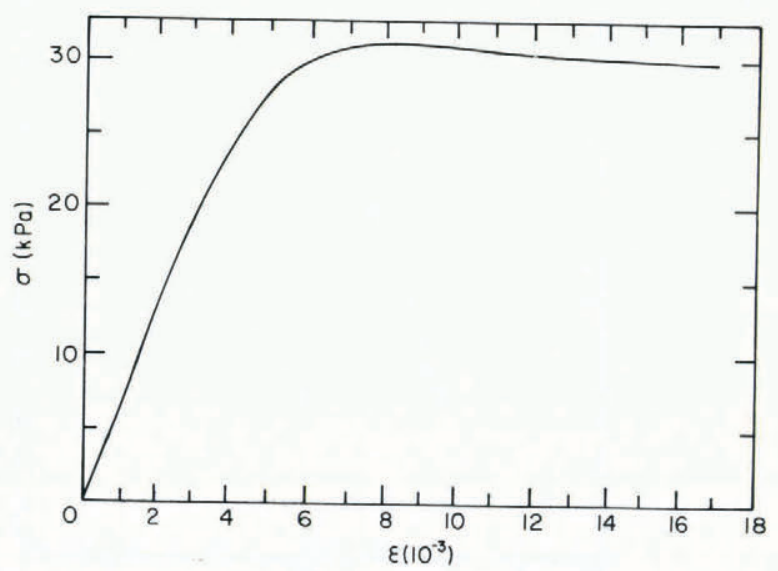
Fig. 3. Experimentally determined stress-strain response for a snow sample strained at a constant tensional rate of
$16.7 \times 10^{-6} s^{-1}$ at $-10^{\circ} \mathrm{C}$. The value of $d \sigma / d \epsilon$ is obtained from this curve.

To obtain the solution, the integral in Equation (II) is numerically integrated using tabulated values of the derivative $\mathrm{d} \sigma / \mathrm{d} \epsilon$ from an experimentally-determined stress-strain response curve obtained from a test at a constant strain-rate of $1.67 \times 10^{-5} \mathrm{~s}^{-1}$ (Fig. 3). To obtain the pattern of the acoustic emission response, we have taken the value of $K$ in Equation (I I) to be zero. This assumes that the low-stress potential for acoustic activity is small compared with the value generated by the integral. The various values associated with Equations (5) and (8) are taken from Table I for a strain-rate of $1.67 \times 10^{-5} \mathrm{~s}^{-1}$. The experimentally-observed acoustic emission response and the response described by Equation (I I) are exhibited in Figure 4. The circles in Figure 4 represent the experimentally observed
points.

The constant strain-rate test is a complex test to interpret. The constant load test (creep test) is somewhat easier. In the creep experiment the value of the integral in Equation (I I) is 


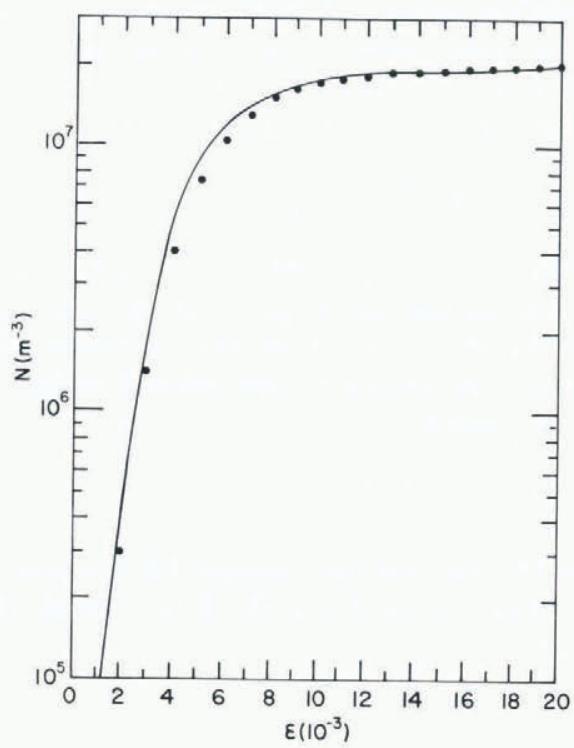

Fig. 4. Total number $\mathcal{N}$ of acoustic emissions per unit volume as a function of strain for a snow sample strained at a constant tensional rate of $16.7 \times 10^{-6} \mathrm{~s}^{-1}$ at $10^{\circ} \mathrm{C}$. The circles represent experimental data points and the curve is the response as determined from Equation (II).

constant after the application of the load. This is because $\mathrm{d} \sigma / \mathrm{d} \epsilon$ in that equation is zero after the load is applied. For a creep test conducted over long periods of time, we can write

$$
\mathcal{N}_{0}=K+\int \exp \left\{\frac{c}{\phi} \exp (-\phi \epsilon)\right\} \frac{\partial \mathcal{N}}{\partial \sigma} \frac{\mathrm{d} \sigma}{\mathrm{d} \epsilon} \mathrm{d} \epsilon,
$$

so that Equation (I I) reduces to

$$
\mathcal{N}=\mathcal{N}_{0} \exp \left\{-\frac{c}{\phi} \exp (-\phi \epsilon)\right\} .
$$

Thus we have solutions for both the constant strain-rate test and the constant load test.

\section{Discussion}

In this paper a model is presented that is intended to describe the acoustic emission response in snow. We have also made the assumption that the acoustic emission activity is a measure of the creep rupture activity in snow. Thus we have attempted to relate the pattern of ultrasonic emissions observed to the fracture phenomenon through stress and strain parameters. This model will undoubtedly undergo further refinement so that we rely less on phenomenological descriptions and more on physically oriented descriptions as we come to understand more of the fundamental nature of the processes involved. One possible application of this analysis, in the field of avalanche mechanics, is to describe several avalanche "release mechanisms".

As indicated in the Introduction, ultrasonic emissions monitored in situ in cold snow generally indicate stability. One instance where this may not be true is where avalanche release is associated with a tertiary (accelerating) creep phenomenon. In terms of the model presented in this paper, so-called "post-control release avalanches" can be rationalized by considering Equations (12) and (13). If by artificial or natural means the stress on a snow 
slope is raised above some critical value and held constant at that value, it is possible for a major failure to take place after some period of time. In terms of Equation (12), if the number $\mathcal{N}_{0}$ of potential fracture sites at a given stress level exceeds some critical number $\mathcal{N}$ of bonds necessary for material integrity, then material failure will occur when

$$
\mathcal{N}_{0} \exp \left\{-\frac{c}{\phi} \exp (-\phi \epsilon)\right\}>\mathcal{N},
$$

at some critical value of strain $\epsilon$. This may also be the case for the long-term crown failures associated with glide slabs. It should be noted that we have used the same symbols for acoustic emissions per unit volume $\left(\mathcal{N}, \mathcal{N}_{0}\right)$ as for the localized internal failures of the snow. If we consider that the acoustic emissions are proportional to the internal fractures, this tacit assumption is very likely to be correct.

\section{Acknowledgements}

I thank Dr Charles Bradley, Dr Robert Brown, Dr Samuel Colbeck, and Dr Richard Sommerfeld for their helpful criticisms of this work. I also thank Ms Mona McDonald for her assistance in editing this paper.

\section{REFERENCES}

Bowles, D., and St Lawrence, W. F. 1977. Acoustic emissions in the investigation of avalanches. Proceedings of the Western Snow Conference, 45th annual meeting, p. 88-94.

Gold, L. W. 196o. The cracking activity of ice during creep. Canadian Journal of Physics, Vol. 38, No. 9, p. $1137-148$.

Gold, L. W. 1972[a]. The failure process in columnar-grained ice. Canada. National Research Council. Division of Building Research. Technical Paper No. 369 .

Gold, L. W. $1972[\mathrm{~b}]$. The process of failure of columnar-grained ice. Philosophical Magazine, Eighth Ser., Vol. 26, No. 2, p. 311-28.

Gubler, H. 1979. Acoustic emission as an indication of stability decrease in fracture zones of avalanches. Fournal of Glaciology, Vol. 22, No. 86, p. 186-88.

St Lawrence, W. F. r 979 . A phenomenological description of the acoustic emission response in several polycrystalline materials. Fournal of Testing and Evaluation, Vol. 7, No. 4, p. 223-28.

St Lawrence, W. F. Unpublished. A structural theory for the deformation of snow. [Ph.D. thesis, Montana State University, Bozeman, 1977.]

St Lawrence, W. F., and Bradley, C. C. [1975.] The deformation of snow in terms of a structural mechanism. [Union Géodésique et Géophysique Internationale. Association Internationale des Sciences Hydrologiques. Commission des Neiges et Glaces.] Symposium. Mécanique de la neige. Actes du colloque de Grindelwald, avril 1974, p. 155-70. (IAHSAISH Publication No. I 14.)

St Lawrence, W. F., and Bradley, C. C. 1977. Spontaneous fracture initiation in mountain snow-packs. Journal of Glaciology, Vol. 19, No. 81, p. 41 1-1 7.

Sommerfeld, R. A. 1977. Preliminary observations of acoustic emissions preceding avalanches. Fournal of Glaciology, Vol. 19, No. 81, p. 399-409. 